\title{
BMJ Open Use of the Safewards Model in healthcare services: a mixed-method scoping review protocol
}

\author{
Marie Gerdtz (D) , Catherine Daniel (D) , Rebecca Jarden (D) , Suzanne Kapp
}

To cite: Gerdtz M, Daniel C, Jarden $\mathrm{R}$, et al. Use of the Safewards Model in healthcare services: a mixed-method scoping review protocol. BMJ Open 2020;10:e039109. doi:10.1136/ bmjopen-2020-039109

- Prepublication history and additional material for this paper are available online. To view these files, please visit the journal online (http://dx.doi. org/10.1136/bmjopen-2020039109).

Received 13 April 2020 Revised 13 November 2020 Accepted 18 November 2020

D) Check for updates

(c) Author(s) (or their employer(s)) 2020. Re-use permitted under CC BY-NC. No commercial re-use. See rights and permissions. Published by BMJ.

Nursing, The University of Melbourne, Melbourne, Victoria, Australia

Correspondence to

Dr Catherine Daniel;

daniel.c@unimelb.edu.au

\section{ABSTRACT}

Introduction Safewards is an organisational approach to delivering inpatient mental health services. The aim of Safewards is to minimise the number of situations in which conflict arises between healthcare workers and patients that lead to the use of coercive interventions (restriction and/or containment).

The Safewards Model has been developed, implemented and evaluated for its impact on all forms of containment. Safewards has been adopted as the recommended approach to preventing patient agitation and clinical aggression in some jurisdictions. Notwithstanding these recommendations, the outcomes of Safewards for staff and patients have not been comprehensively described. The aim of the scoping review is to describe (1) Safewards interventions; (2) how Safewards interventions have been implemented in healthcare settings; (3) outcome measures used to evaluate the effectiveness of Safewards; (4) barriers and enablers to the uptake and sustainability of Safewards. This review will provide a foundation for further research and/or systematic review of the effectiveness of Safewards.

Methods and analysis Peer-reviewed manuscripts of quantitative, qualitative and mixed-method research in English with be included for the period 01 January 2013-December 31st 2020. Electronic databases including Cumulative Index to Nursing and Allied Health Literature, Cochrane, Embase, Emcare, Joanna Briggs Institute, Medline, Global Health, PsycINF0 and Scopus will be searched. Preferred Reporting Items for Systematic Reviews and Meta-Analysis extension for Scoping Reviews checklist and explanation and the Preferred Reporting Items for Systematic Reviews and Meta-Analysis Protocol will be followed. Publications will be excluded if they do not include the required participants, concept or context. Two reviewers will independently screen all titles and abstracts and full-text studies for inclusion.

Ethics and dissemination Ethical approval for this review is not required as the information to be collected is publicly available. There are no participants or safety considerations in this review of published literature. Key findings for future research and clinical practice will be disseminated though peer-reviewed publication, stakeholder reporting and conference presentations.

\section{BACKGROUND}

Accessing healthcare can be a challenging and stressful experience for patients and their

\section{Strengths and limitations of this study}

This scoping review protocol is the first to focus on the Safewards interventions using a transparent and systematic method to reduce bias.

- The comprehensive search strategy, inclusive of grey literature, will support the identification of a broad range of interprofessional publications.

- The specified data extraction fields will support the identification of effectiveness, barriers and enablers, but this review does not seek to establish effectiveness.

- The scoping review will synthesise findings across a broad range of study types and designs.

- Use of the Preferred Reporting Items for Systematic Reviews and Meta-Analyses extension for Scoping Reviews tool will support a systematic approach to the reporting.

carers/families. This is particularly so when a person is experiencing sudden ill health or injury, pain, discomfort and diagnostic uncertainty. The complexity of healthcare environments and healthcare systems can further contribute to suboptimal communication resulting in conflict between patients and healthcare providers. Such situations can result in aggressive and/or violent behaviours by patients that may serve as precipitants to further negative actions by the patient. This includes patients self-harming or deciding to leave part-way through treatment or against medical advice. These actions expose healthcare organisations to risk and individual staff to potential injury. ${ }^{1}$

To maintain the safety of patients and healthcare workers, restrictive interventions, specifically physical, mechanical and chemical restraint, may be employed alone or in combination. ${ }^{23}$ The use of restrictive interventions in acute healthcare settings, including hospital emergency departments (EDs), is common practice. ${ }^{4}$ Despite this, there are significant harms associated with restriction. ${ }^{5}$ Research shows that restrictive interventions used in the context of a "code 
grey' response ${ }^{6}$ (emergency response from security and clinical staff to an unarmed threat) are associated with negative experience and emotions among both patients and staff. ${ }^{78}$

The use of restrictive interventions disrupts the therapeutic relationship between the patient, healthcare workers and their families. This, in turn, negatively impacts the ability of the patient and healthcare professional to work cooperatively to optimise health and wellbeing. ${ }^{9}$ The adverse effects of restrictive interventions are particularly detrimental for those people who may suffer long-term mental health conditions and need to access healthcare in the future. Importantly authors have noted that previous use of restrictive interventions influences patient decisions to seek subsequent hospital care.$^{10}$ Physical restraint continues to be used despite known physical and/or psychological harm experienced by mental health patients; international concern over that restraint still occurs, as well as the call for eradication of this practice. ${ }^{9}$

The Safewards Model is a way of organising and delivering health services to reduce situations where conflict during interactions with patients may lead to containment (such as seclusion in a locked room or the use of physical and/or mechanical restraint). ${ }^{1}$ The Safewards Model was developed initially for use in mental health services; however, it has since been used to prevent conflict and containment in other healthcare settings. Safewards presents a way for healthcare staff to identify and address triggers resulting in conflict by, for example, staff imposing rules and restrictions on patient and family contact. Staff actions may, at times, serve as antecedents to conflict and the subsequent use of harmful interventions (such as seclusion and restraint). Ultimately Safewards aims to reduce the likelihood of conflict and containment. ${ }^{11}$

Safewards is the recommended model of care in Australia for preventing agitation and aggression among patients ${ }^{12}$ and is so regarded internationally. ${ }^{13}$ The Safewards Model according to Bowers employs a set of prevention and intervention strategies that are designed to promote a therapeutic response to minimise conflict and containment among patients thereby optimising patient and staff safety. ${ }^{14}$

The Safewards Model requires healthcare providers who are educated and skilled in this approach to recognise contributing factors that lead to aggression and conflict and then respond to reduce the risk of situational triggers that may result in containment. ${ }^{15} 16$ Where a restrictive intervention is deemed necessary, on the basis of patient safety, the Safewards Model advocates the application of least restrictive intervention/s to prevent harm and minimise conflict. ${ }^{11}$

There has been extensive funding allocated to reducing the risk of violence in healthcare both internationally and nationally. For example, in Australia there are initiatives funded by work compensation insurance schemes to increase public awareness of violence in healthcare. ${ }^{17}$ Likewise strategies to improve ward and hospital security through staff training and security reviews have been implemented. ${ }^{18} 19$ These initiatives, however, do not address the factors that contribute to episodes of violence, or provide evidence of the best interventions to ensure staff and patient safety.

There are several organisational initiatives to reduce occupational violence and the use of restrictive interventions. One example is the Six Core Strategies programme which uses data, leadership, workforce development, prevention strategies such as de-escalation, enhancing consumer roles and debriefing in mental health settings to reduce the use of seclusion and restraint. ${ }^{20}$ This model has been successful in reducing restraint and seclusion in mental health settings and highlights the need to understand the dynamic relationship between health services, model implementation and consumer outcomes in different settings. ${ }^{21}$

A preliminary search of the literature identified some studies reporting favourable outcomes for patients, healthcare providers and systems when healthcare services employ the Safewards Model. Internationally, there has been one randomised controlled trial in adult mental health inpatient wards $(\mathrm{n}=16)$ that found a significant reduction in rates of conflict by $15 \%$ (95\% CI $5.7 \%$ to $23.7 \%$ ) and containment by $23.2 \%(95 \%$ CI $9.9 \%$ to $35.5 \%)$ relative to control wards $(\mathrm{n}=15) .{ }^{14}$ In Australia, a before and after comparison study of the effect of Safewards on the practices of nurses in 13 mental health inpatient units identified a $36 \%$ reduction in seclusion incident rate ratio (from 1.0 to $0.64, \mathrm{p}=0.04$ ) at 12 -month follow-up in wards using Safewards, compared with no change in comparison wards. ${ }^{22}$ This evaluation also reported that implementation of Safewards improved communication, optimism and relationships among patients and healthcare providers.

Research has shown that increasing awareness through collaboration and reflection by ED nurses leads to a shift in attitudes towards restraint with less reliance on coercive interventions. ${ }^{23}$ Despite this, restrictive interventions used in EDs continue and only a minority of people who experience restraint are admitted to mental health wards. $^{4}$

Given the prevalence, cost of conflict and containment, and negative outcomes for patients and staff, there is potential for the Safewards Model to reduce situations where conflict during interactions with patients may lead to containment.

The aim of the scoping review is to comprehensively describe:

1. Safewards interventions.

2. How Safewards interventions have been implemented in healthcare settings.

3. Outcome measures that have been used to evaluate the effectiveness of Safewards.

4. Barriers and enablers to the implementation, uptake and sustainability of Safewards as reported by patients, staff and researchers.

It is anticipated that the scoping review will be useful for health services considering implementation of Safewards 
and will establish the need, or otherwise, for further research and/or systematic review of the effectiveness of the Safewards Model on reducing the rates of conflict and containment and/or the experiences of healthcare staff and patients. The review will describe the Safewards Model and components implemented to answer the following three questions:

1. What Safewards interventions have been evaluated and subsequently reported in published peer-reviewed research literature?

2. Which outcome measures have been used to evaluate the impact of the Safewards Model in practice?

3. What are the barriers and enablers to implementation of the Safewards Model?

\section{METHOD}

The scoping review framework has been adapted from the work of Arksey and O'Malley, ${ }^{24}$ Levac et $a l^{25}$ and Peters $e t$ $a l .{ }^{26}$ The current review protocol integrates these frameworks with the Preferred Reporting Items for Systematic Reviews and Meta-Analysis extension for Scoping Reviews checklist and explanation. ${ }^{27}$ This approach is aligned with the Preferred Reporting Items for Systematic Reviews and Meta-Analysis Protocol (PRISMA-P). ${ }^{28}$ The Joanna Briggs Institute Reviewer's Manual ${ }^{29}$ will guide a stepwise approach. The review title was registered with The Joanna Briggs Institute on the 5th of February 2020.

\section{Ethics and dissemination}

Ethical approval for this review is not required as the information to be collected is publicly available. There are no participants or safety considerations in this review of published literature. Key findings for future research and clinical practice will be disseminated through peerreviewed publication, stakeholder reporting and conference presentations.

\section{Patient and public involvement}

Patients and the public were not involved in the design nor planning of this scoping review.

\section{Inclusion criteria \\ Types of participants}

Patients, carers (formal and informal), accompanying persons and healthcare professionals.

\section{Concept}

Safewards Model and interventions, measures, outcomes, barriers and enablers. The paper will report all outcome measures, including those not yet validated, for studies that investigate effectiveness.

\section{Context}

Any healthcare service/s: mental health, inpatient units, forensic mental health, ED, acute health.

\section{Types of publications}

Peer-reviewed journal publications of quantitative, qualitative and mixed-method primary research published in
English from 1st January 2013 to 31st December 2020. This period was chosen because the Safewards Model was first evaluated in 2013. Grey literature will be included to capture other reports of the implementation of Safewards that have not been published in academic literature.

\section{Search strategy}

We will search electronic databases including Cumulative Index to Nursing and Allied Health Literature (CINAHL), Cochrane, Embase, Emcare, Joanna Briggs Institute, Medline, Global Health, PsycINFO and Scopus. Grey literature will be searched using OpenDissertations, OpenGrey and Trove. Two reviewers will independently screen all titles and abstracts, and full-text studies, for inclusion according to the predetermined inclusion and exclusion criteria. The search terms will include the following keywords (and associated index terms): Safewards', 'Safe-wards' and 'Safe+wards'.

To identify potentially relevant publications, a threestage search process will be executed. First, the search strategy will be tested in two electronic databases (CINAHL and Medline) and we will identify additional relevant keywords and index terms. Additionally, we will conduct a search of keywords and index terms in all the included databases. Finally, we will review the reference lists of included studies to identify any additional relevant publications. The proposed search strategy for CINAHL and Medline is presented in online supplemental appendix 1.

\section{Study selection}

To achieve consistency among reviewers, the first two included publications will be independently screened (according to the inclusion and exclusion criteria) by all reviewers (authors 1, 2, 3 and 4) and the process and results discussed before continuing with the review. Thereafter, two reviewers will independently screen the title and abstracts of the publications identified in the search followed by full-text screening of those potentially meeting the inclusion criteria. Disagreements on study inclusion will be resolved through discussion and consensus, or a third reviewer.

\section{Data extraction}

Data from eligible studies will be extracted independently by two reviewers. Extracted data will be recorded in an extraction tool in Microsoft Excel which has been purposely designed for the study (online supplemental appendix 2). The tool will record publication details, theoretical frameworks, research questions, aims and objectives, design, sample, setting, interventions, outcomes and measures used. A 'Template for Intervention Description and Replication checklist ${ }^{30}$ will be completed for each intervention.

Presentation of the results

Results of the search strategy and selection process (number of citations, titles and abstracts, full-text articles, included and excluded studies) will be presented 
in a PRISMA flow chart with a narrative summary. ${ }^{31} \mathrm{~A}$ synthesis of the study results according to participants, concept and context will be presented in tables and diagrams with accompanying narrative summaries. For both interventional studies and qualitative research, we will provide a table of results including year published, country of origin, date of research, Safewards interventions implemented, measures of effectiveness, and barriers and enablers to implementation. A convergentsegregated approach will be used to extract and analyse quantitative and qualitative data simultaneously.

Contributors $M G$ and $C D$ established the need for a scoping review. MG, $C D, R J$ and SK drafted the initial protocol. All authors have reviewed and contributed to the manuscript.

Funding The authors have not declared a specific grant for this research from any funding agency in the public, commercial or not-for-profit sectors.

Competing interests None declared.

Patient consent for publication Not required.

Provenance and peer review Not commissioned; externally peer reviewed.

Supplemental material This content has been supplied by the author(s). It has not been vetted by BMJ Publishing Group Limited (BMJ) and may not have been peer-reviewed. Any opinions or recommendations discussed are solely those of the author(s) and are not endorsed by BMJ. BMJ disclaims all liability and responsibility arising from any reliance placed on the content. Where the content includes any translated material, BMJ does not warrant the accuracy and reliability of the translations (including but not limited to local regulations, clinical guidelines, terminology, drug names and drug dosages), and is not responsible for any error and/or omissions arising from translation and adaptation or otherwise.

Open access This is an open access article distributed in accordance with the Creative Commons Attribution Non Commercial (CC BY-NC 4.0) license, which permits others to distribute, remix, adapt, build upon this work non-commercially, and license their derivative works on different terms, provided the original work is properly cited, appropriate credit is given, any changes made indicated, and the use is non-commercial. See: http://creativecommons.org/licenses/by-nc/4.0/.

\section{ORCID iDs}

Marie Gerdtz http://orcid.org/0000-0002-2100-994X

Catherine Daniel http://orcid.org/0000-0002-1122-3950

Rebecca Jarden http://orcid.org/0000-0003-4643-7147

Suzanne Kapp http://orcid.org/0000-0002-5438-8384

\section{REFERENCES}

1 Bowers L, Alexander J, Bilgin H, et al. Safewards: the empirical basis of the model and a critical appraisal. J Psychiatr Ment Health Nurs 2014;21:354-64.

2 Downes MA, Healy P, Page CB, et al. Structured team approach to the agitated patient in the emergency department. Emerg Med Australas 2009;21:196-202.

3 Medley DB, Morris JE, Stone CK, et al. An association between occupancy rates in the emergency department and rates of violence toward staff. J Emerg Med 2012;43:736-44.

4 Knott J, Gerdtz M, Dobson S, et al. Restrictive interventions in Victorian emergency departments: a study of current clinical practice. Emerg Med Australas 2020;32:393-400.

5 Yap CYL, Taylor DM, Kong DCM, et al. Risk factors for Sedationrelated events during acute agitation management in the emergency department. Acad Emerg Med 2019;26:1135-43.

6 State Government of Victoria. Code grey standards, 2017. Available: https://www2.health.vic.gov.au/healthworkforce/ worker-healthwellbeing/occupational-violence-aggression/code-grey-black
7 El-Badri S, Mellsop G. Patient and staff perspectives on the use of seclusion. Australas Psychiatry 2008;16:248-52.

8 Moran A, Cocoman A, Scott PA, et al. Restraint and seclusion: a distressing treatment option? J Psychiatr Ment Health Nurs 2009;16:599-605.

9 Cusack P, Cusack FP, McAndrew S, et al. An integrative review exploring the physical and psychological harm inherent in using restraint in mental health inpatient settings. Int $J$ Ment Health Nurs 2018;27:1162-76.

10 Currier GW, Walsh P, Lawrence D. Physical restraints in the emergency department and attendance at subsequent outpatient psychiatric treatment. J Psychiatr Pract 2011;17:387-93.

11 Bowers L. Safewards: a new model of conflict and containment on psychiatric wards. J Psychiatr Ment Health Nurs 2014;21:499-508.

12 Australian Commission on Safety and Quality in Health Care. The National safety and quality health service (NSQHS) standards, 2019. Available: https://www.safetyandquality.gov.au/standards/nsqhsstandards/comprehensive-care-standard/minimising-patient-harm/ action-533 [Accessed 9 Jan 2020].

13 Baumgardt J, Jäckel D, Helber-Böhlen $\mathrm{H}$, et al. Preventing and reducing coercive Measures-An evaluation of the implementation of the Safewards model in two locked wards in Germany. Front Psychiatry 2019;10:340.

14 Bowers L, James K, Quirk A, et al. Reducing conflict and containment rates on acute psychiatric wards: the Safewards cluster randomised controlled trial. Int J Nurs Stud 2015;52:1412-22.

15 Duxbury J. An exploratory account of registered nurses' experience of patient aggression in both mental health and general nursing settings. J Psychiatr Ment Health Nurs 1999;6:107-14.

16 Duxbury J, Whittington R. Causes and management of patient aggression and violence: staff and patient perspectives. J Adv Nurs 2005;50:469-78.

17 Victorian WorkCover Authority. Prevention and management of violence and aggression in health services, 2017.

18 State Government of Victoria. Guide for security arrangements to prevent and manage occupational violence and aggression: guiding principles, 2018. Available: https://www2.health.vic.gov.au/about/ publications/policiesandguidelines/guide-for-security-arrangementsto-prevent-manage-ova

19 Minister of Health. More funds to keep our health workers safe at work, 2018. Available: https://www.premier.vic.gov.au/more-fundsto-keep-our-health-workers-safe-at-work/

20 Huckshorn KA. Reducing seclusion restraint in mental health use settings: core strategies for prevention. J Psychosoc Nurs Ment Health Serv 2004;42:22-33.

21 Wieman DA, Camacho-Gonsalves T, Huckshorn KA, et al. Multisite study of an evidence-based practice to reduce seclusion and restraint in psychiatric inpatient facilities. Psychiatr Serv 2014;65:345-51.

22 Fletcher J, Spittal M, Brophy L, et al. Outcomes of the Victorian Safewards trial in 13 wards: impact on seclusion rates and fidelity measurement. Int J Ment Health Nurs 2017;26:461-71.

23 Gerdtz MF, Daniel C, Dearie V, et al. The outcome of a rapid training program on nurses' attitudes regarding the prevention of aggression in emergency departments: a multi-site evaluation. Int J Nurs Stud 2013;50:1434-45.

24 Arksey H, O'Malley L. Scoping studies: towards a methodological framework. Int J Soc Res Methodol 2005;8:19-32.

25 Levac D, Colquhoun H, O'Brien KK. Scoping studies: advancing the methodology. Implement Sci 2010;5:69.

26 Peters MDJ, Godfrey CM, Khalil H, et al. Guidance for conducting systematic scoping reviews. Int J Evid Based Healthc 2015;13:141-6.

27 Tricco AC, Lillie E, Zarin W, et al. PRISMA extension for scoping reviews (PRISMA-ScR): checklist and explanation. Ann Intern Med 2018;169:467-73.

28 Moher D, Shamseer L, Clarke M, et al. Preferred reporting items for systematic review and meta-analysis protocols (PRISMA-P) 2015 statement. Syst Rev 2015;4:1.

29 Aromataris E, Munn Z. Joanna Briggs Institute Reviewer's Manual. The Joanna Briggs Institute, 2017.

30 Hoffmann TC, Glasziou PP, Boutron I, et al. Better reporting of interventions: template for intervention description and replication (TIDieR) checklist and guide. BMJ 2014;348:g1687.

31 Moher D, Liberati A, Tetzlaff J, et al. Preferred reporting items for systematic reviews and meta-analyses: the PRISMA statement. PLoS Med 2009;6:e1000097. 